\title{
Implementation of the Walrasian correspondence by market games
}

\author{
Carmen Beviá $^{1}$, Luis C. Corchón ${ }^{2}$, Simon Wilkie $^{3}$
}

1 Departament d'Economia i d'Història Econòmica and CODE Universitat Autònoma de Barcelona, Edifici B, 08193 Bellaterra (Barcelona), Spain (e-mail: carmen.Bevia@uub.es)

2 Departamento de Economía. Universidad Carlos III Madrid, Calle Madrid 126, 28903 Getafe (Madrid), Spain (e-mail: chorchon@eco.uc3m.es)

3 Division of Humanities and Social Sciences. California Institute of Technology, Caltech, Pasadena CA 91125, USA (e-mail: wilkie@hss.caltech.edu)

Received: 25 December 1998 / Accepted: 8 April 2002

\begin{abstract}
In this paper we present a set of axioms guaranteeing that, in exchange economies with or without indivisible goods, the set of Nash, Strong and active Walrasian Equilibria all coincide in the framework of market games.
\end{abstract}

\section{Introduction}

In this paper we present an unifying framework for the study of market games in exchange economies. In classical exchange economies when all goods are divisible particular market games have been considered by Shubik (1977) and Schmeidler (1980). A general axiomatic framework was introduced by Benassy (1986). He proposed a set of axioms that defines a market game, and he was able to show that 1) the Walrasian allocation can be supported as a Nash Equilibrium (NE in the sequel), and 2) that when all markets are active, all NE yield Walrasian allocations. It was shown in specific models by Dubey (1982) (assuming divisible goods) and Svensson (1991) (assuming one indivisible good) that the set of Strong Equilibrium (SE) outcomes coincides with the set of NE outcomes when all the markets are active and with the set of Walrasian allocations (other models of indivisibilities include those of Roth 1982; Kaneko 1983; Gale 1984; Quinzii 1984; Svensson 1984, 1988; Demange and Gale 1985; Maskin 1987; Roth and Sotomayor 1988; Tadenuma and Thomson 1990).

\footnotetext{
We are very grateful to William Thomson for many suggestions. Also we would like to thank comments from J. Alcalde, R. Bhattacharya, C. Herrero, P. Marhuenda, D. Pérez-Castrillo, A. RomeroMedina, T. Shinotsuka, T. Sjöström, and A. Villar, an associated editor and an anonymous referee. The first author thanks financial support from DGCYT and Direcció General de Reserca under projects $P B 98$ - 0870, and SGR2000-00054. The second author wishes to acknowledge financial help from DGCYT under project $P B 98-0940$.
} 
A natural question is whether the results explained above depend on either the special structure of the commodity space or on the particular game form used. In this paper we address this question and show that neither is the case. We present a model in which the commodity space has no special structure (and thus it could have both many divisible and many indivisible goods), and we propose a set of axioms that a market game should satisfy. With these axioms in hand we prove that the set of Walrasian allocations, SE outcomes and NE outcomes when all markets are active coincide.

The main insight of this paper is that from the point of view of implementation in NE and SE, convexity does not matter very much. Other insights gained by the study of market games are the following: 1) To sustain Walrasian allocations as NE or SE equilibria outcomes requires relatively harmless and simple assumptions. 2) However, to eliminate non-Walrasian equilibria requires strong assumptions on the mechanism (i.e., some kind of Bertrand competition which implies that the outcome function is discontinuous, see Benassy 1986, pp.99-100) and the assumption that all markets are active. 3) Individual feasibility cannot be guaranteed outside equilibrium. ${ }^{1}$ And 4 ) as long as all markets are active, the fact that coalitions can or can not be formed does not matter since implementation occurs in NE and SE. Given that Implementation by means of market games has intuitive appeal because the underlying mechanism is both natural and simple, it would be nice to know if our results can be extended to production economies and other social choice rules.

Our work can be regarded as an application of implementation theory to a specific problem. The authors hope that it might modestly contribute to show that implementation is not only about abstruse mechanisms but can also shed some light on the functioning of real life institutions like double auction markets. It should be remarked though that market games mimic but are not markets (in the same way than an artificial heart mimics but is not a heart). In particular some tendencies that are spontaneous in a market are reflected here in the form of the outcome function. For instance, in a frictionless market arbitrage implies that there is only one trading price for each good. In the class of market games considered here, the existence of an unique trading price is assumed directly.

The rest of the paper goes as follows. In the next section we present our model of a market game. Section 3 gathers our main results. Finally, Sect. 4 offers some comments.

\section{The model}

This section has five parts. a) The description of the environment, b) the definition of a social choice correspondence, $c$ ) the description of a game form (also called a mechanism), d) the definition of the game-theoretical equilibrium concepts and e) the notion of implementation.

\footnotetext{
${ }^{1}$ For the issue of Individual Feasibility see our comments after Theorem 1. See also Postlewaite and Wettstein (1989).
} 


\subsection{Environment}

There are $h$ consumers and $n+1$ goods, where $n$ and $h$ are natural numbers. We denote by $H$ the set of consumers. Goods $1, \ldots, n$ are (possibly indivisible) goods, the set of these goods is denoted by $G$. Good 0 is a perfectly divisible good which can be loosely termed as money. A net trade for consumer $i$ is an $n+1$ dimensional vector $\left(x_{i 1}, \ldots, x_{i n}, m_{i}\right)$, sometimes also represented by $\left(x_{i}, m_{i}\right)$, where $x_{i g}$ (resp. $m_{i}$ ) is her net trade of commodity $g \in G$ (resp. money). The set of individually feasible net trades for consumer $i$ is denoted by $X_{i}=C_{i} \times M_{i}$ where $x_{i} \in C_{i}$ and $m_{i} \in M_{i}=\mathbb{R}$. We do not impose any structure on $C_{i}$ except that $C_{i}$ is a subset or $R^{n}$. The preferences of $i$ over net trades in $X_{i}$ are represented by a utility function $u_{i}: X_{i} \rightarrow \mathbb{R}$ denoted by $u_{i}=u_{i}\left(x_{i}, m_{i}\right)$ which is assumed to be strictly increasing in money. We will also assume that any bundle inside $X_{i}$ is preferred to any bundle outside $X_{i}{ }^{2}$ and that $u_{i}$ is never decreasing in any of its arguments, i.e., goods can be freely disposable by consumers. Let us denote by $(x, m) \in R^{(n+1) h}$ the $h$-tuple of net trades for consumers $1, \ldots, h$ also referred to as an allocation. An allocation $(x, m)$ is said to be balanced if $\forall g \in G, \sum_{i \in H} x_{i g} \leq 0$ and $\sum_{i \in H} m_{i} \leq 0$. We remark that a balanced allocation does not need to be totally feasible because agents may receive net trades which are not individually feasible. The set of balanced allocations is denoted by $F$. We will assume that the number of agents and the individually feasible net trade sets are fixed. Thus an economy, denoted either by $e$ or by $\left(u_{i}\right)_{i \in H}$, is a list of utility functions, one for each agent. Let us denote by $\mathcal{E}$ the set of admissible economies.

\subsection{Social choice correspondence}

A social choice correspondence $\Phi: \mathcal{E} \rightarrow F$ associates a non-empty set of balanced allocations to every $e \in \mathcal{E}$. It is meant to represent the objectives of the society. In this paper $\Phi$ will be the Walrasian correspondence. In order to define this let us first define a Walrasian Equilibrium (WE in the sequel) for a particular economy $e \in \mathcal{E}$.

Definition 1. A Walrasian Equilibrium for an economy $\left(u_{i}\right)_{i \in H}$ is a balanced allocation $\left(x^{w}, m^{w}\right)$ and a vector of prices $p^{w} \in \mathbb{R}_{+}^{n}$ such that $\forall i \in H$

$$
\left(x_{i}^{w}, m_{i}^{w}\right) \text { maximizes } u_{i}\left(x_{i}, m_{i}\right) \text { subject to } p^{w} x_{i}+m_{i}=0, .
$$

Notice that in the above definition money is the numeraire. The Walrasian social choice correspondence maps the set of economies in allocations that are Walrasian equilibria for these economies. In what follows we assume that the domain $\mathcal{E}$ is such that the Walrasian social choice correspondence is well defined.

2 This assumption is needed to evaluate bundles outside consumption sets, see Subsect. 2.3 below. A similar assumption is made by Hurwicz (1979 b), Schmeidler (1980) and Benassy (1986). 


\subsection{Game forms and market games}

A Game Form (or a mechanism) is a list $\left(S_{i}, f\right), i \in H$, where $S_{i}$ denotes the strategy set of consumer $i$ and $f$ is the outcome function. In a market game a strategy for consumer (player) $i$ is a $2 n$ dimensional vector of real numbers $s_{i}=\left(\pi_{i}, q_{i}\right)$ where $\pi_{i}=\left(\pi_{i 1}, \ldots, \pi_{i n}\right)$ (resp. $\left.q_{i}=\left(q_{i 1}, \ldots, q_{i n}\right)\right)$ represents the vector of bids (resp. net trades) proposed by player $i$. Let us denote by $\pi$ (resp. by $q$ ) the list of bids (resp. net trades) proposed by all players and by $s \equiv(\pi, q) \in \mathbb{R}^{2 n h}$ the list of all strategies for all players. Let $S \equiv \prod_{i \in H} S_{i}$. The outcome function $f$ maps $S$ into the set of balanced allocations, i.e., $f: S \rightarrow F$. This implies that we do not require allocations to be individually feasible: Bundles outside the consumption set are evaluated according with the extension of preferences made in part a) above. ${ }^{3}$ Let $f_{i}$ be the $i^{\text {th }}$ component of $f$. It represents the allocation of goods and money obtained by $i$ i.e., $f_{i}(s) \equiv\left(x_{i}(s), m_{i}(s)\right)$. Let $p$ be the $n$-dimensional vector of trading prices as a function of $s$, i.e., $p: S \rightarrow R_{+}^{n}$ or $p=p(s) .{ }^{4}$ The vector $p$ is such that $\sum_{i \in H} x_{i g}<0$ implies $p_{g}=0$. The budget constraint for consumer $i$ is $B_{i}(p)=\left\{\left(x_{i}, m_{i}\right) \in X_{i} \mid p x_{i}+m_{i} \leq 0\right\}$. The outcome function $f$ selects allocations satisfying the budget constraint of each consumer with equality, and thus the quantity of money allocated to consumer $i$, denoted by $m_{i}(s)$, is $m_{i}(s)=-p(s) x_{i}(s)$, where $x_{i}(s)$ is the net trade of goods allocated to $i$ if the strategy $s$ is played.

\subsection{Equilibrium}

Let $v_{i}(s) \equiv u_{i}\left(f_{i}(s)\right)$ be the indirect utility function associated to the list of strategies $s \in \mathrm{S}$. Also let us denote by $s_{-i}$ the list of strategies played by all consumers except $i$. Then we have the following definition:

Definition 2. A Nash Equilibrium (NE) of a game form $(S, f)$ for an economy $\left(u_{i}\right)_{i \in H}$ is a list of strategies $s^{n} \in S$ such that $\forall i \in H$,

$$
v_{i}\left(s^{n}\right) \geq v_{i}\left(s_{i}, s_{-i}^{n}\right) \forall s_{i} \in S_{i} .
$$

A coalition, denoted by $C$, is a non empty subset of the set of all players. A list of strategies for all members of $C$ is denoted by $s_{c}$ and the corresponding strategy set by $S_{c}$. Similarly $s_{-c}$ denotes the list of strategies for all players not in $C$.

Definition 3. A Strong Equilibrium (SE) of a game form $(S, f)$ for an economy $\left(u_{i}\right)_{i \in H}$ is a list of strategies $s^{s} \in S$ such that there is no coalition $C$, and $s_{c} \in S_{c}$ such that $v_{i}\left(s_{c}, s_{-c}^{s}\right) \geq v_{i}\left(s^{s}\right), \forall i \in C$ and $v_{j}\left(s_{c}, s_{-c}^{s}\right)>v_{j}\left(s^{s}\right)$ for some $j \in C{ }^{5}$

\footnotetext{
3 The extension of our results to a mechanism where allocations are always individually feasible is discussed after Theorem 1 below.

${ }^{4}$ Benassy does not assume directly, as we do, that all traders buy at the same price. This possibility can be considered in our model at the cost of some complications. However, under Benassy's assumptions, at any NE their trading prices are identical.

5 An alternative definition of strong equilibrium requires that the inequality be strict for all members of C. In our model both notions coincide.
} 


\subsection{Implementation}

Let $N E(S, f, e)$ be the set of Nash Equilibrium strategies for the mechanism $(S, f)$ and the economy $e$. Let $S E(S, f, e)$ be the set of Strong Equilibrium strategies for the mechanism $(S, f)$ and the economy $e$. Then, we come to the main definitions of the paper.

Definition 4. The Game form $(S, f)$ implements a social choice correspondence $\Phi$ in the domain $\mathcal{E}$ in Nash Equilibrium if $\forall e \in \mathcal{E}$

$$
\Phi(e)=f(N E(S, f, e)) .
$$

Definition 5. The Game form $(S, f)$ implements a social choice correspondence $\Phi$ in the domain $\mathcal{E}$ in Strong Equilibrium if $\forall e \in \mathcal{E}$

$$
\Phi(e)=f(S E(S, f, e)) .
$$

Definitions 4 and 5 refer to the equilibrium concepts that we will use in the remainder of the paper. The notion of NE is relevant in a non cooperative framework, where agents can not engage in binding agreements. The notion of a SE is meant to capture the outcome of a game in which cooperation or at least some kind of coordination is feasible. If the designer does not have a priori information about the feasibility of those agreements, it appears to be desirable for implementation to occur in both scenarios. This is the concept of double implementation, a term coined by Eric Maskin, which we present formally in the next definition.

Definition 6. The Game form $(S, f)$ doubly implements in Nash and Strong equilibrium a social choice correspondence $\Phi$ in the domain $\mathcal{E}$ if $\forall e \in \mathcal{E}$,

$$
\Phi(e)=f(S E(S, f, e))=f(N E(S, f, e)) .
$$

\section{Results}

In this section we gather together our main findings on market games. Let us now present our two first axioms:

Unanimity (U). If $s \equiv(\pi, q) \in S$ is such that $\pi_{i}=\pi_{j}, \forall i, j \in H$, then $p(s)=\pi_{i}$. If in addition $q$ is such that for all $g \in G \sum_{i} q_{i g}=0$ then $x(s)=q$.

Voluntary Trade (VT). $\forall g \in G, \forall i \in H, \forall s \in S$,

$x_{i g}(s)>0$ implies that $p_{g}(s) \leq \pi_{i g}$ and $x_{i g}(s)<0$ implies that $p_{g}(s) \geq \pi_{i g}$.

Unanimity says that if all bids are the same, trading prices must be equal to these bids, and, if in addition the proposed net trade vector is balanced, net trades allocated by the mechanism must be equal to the net trades asked for by consumers. This is a weak property which must be satisfied by any satisfactory model of resource allocation in market economies. The Voluntary Trade axiom says that in order to 
get a positive (resp. negative) net trade of, say, commodity $g$, the trading price of $g$ must be greater than or equal (resp. lower or equal) to the bid of any seller (resp. buyer). Under the VT axiom the interpretation of bids is that if consumer $i$ is a net demander (resp. supplier) of good $g, \pi_{i g}$ is the maximum (resp. minimum) price at which she is prepared to accept a net trade of $q_{i g}$ units of good g. Special cases of this property have been proposed by Benassy (1986, p. 100), Svensson (1991) and Silvestre (1985). Now, we are ready to prove our first result.

Proposition 1. Let $(S, f)$ be a market game form in which VT and U hold. Then, if $\left(p^{w}, x^{w}, m^{w}\right)$ is a WE, $\exists$ s such that $s$ is a SE and $f(s)=\left(x^{w}, m^{w}\right)$.

Proof. Let us first construct s. For a typical consumer i, let $\pi_{i}=p^{w}$ and $q_{i}=x_{i}^{w}$. Then, by $U, f(s)=\left(x^{w}, m^{w}\right)$ and $p^{w}=p(s)$. Therefore if the Proposition were not true there is a coalition $C$, and a $s_{c}^{\prime} \in S_{c}$ such that $v_{i}\left(s_{c}^{\prime}, s_{-c}\right) \geq v_{i}(s), \forall i \in C$ and $v_{j}\left(s_{c}^{\prime}, s_{-c}\right)>v_{j}(s)$ for some $j \in C$. Let $\left(x_{i}^{\prime}, m_{i}^{\prime}\right)=f_{i}\left(s_{c}^{\prime}, s_{-c}\right), \forall i \in C$. Let also $p^{\prime}$ be the new vector of trading prices, i.e., $p^{\prime}=p\left(s_{c}^{\prime}, s_{-c}\right)$. Then by revealed preference we have $p^{w} x_{i}^{\prime}+m_{i}^{\prime} \geq p^{w} x_{i}^{w}+m^{w}=0 \forall i \in C$ and $\exists j \in C$ for whom the inequality is strict.

Since $p^{\prime} x_{i}^{\prime}+m_{i}^{\prime} \leq 0$, it follows from that and the previous inequality that $x_{i}^{\prime}$ $\left(p^{\prime}-p^{w}\right) \leq 0 \forall i \in C$ and $\exists j \in C$ for whom the inequality is strict. Adding over $i$ we get:

$$
\left(p^{\prime}-p^{w}\right) \cdot \sum_{i \in C} x_{i}^{\prime}<0
$$

Since the Walrasian equilibrium is Pareto Efficient, $C$ can not be composed of all agents. Also, if the sum of net demands of the members of $C$ of a good is negative (i.e., $C$ is a net supplier of, say commodity $g$ ) we have two cases. If the complementary coalition is a net demander of $g$, by $V T$ it must be that $p_{g}^{\prime} \leq \pi_{r g}=$ $p_{g}^{w}$ for some $r \notin C$, since all sellers outside the coalition are bidding $p^{w}$. If the complementary coalition is not a net demander of $g, p_{g}^{\prime}=0$. In any case, the corresponding term in (1) above is positive or zero. If the sum of net demands of the members of $C$ of a good $g$ is positive, the complementary coalition is a net supplier of $g$, by $V T$ it must be that $p_{g}^{\prime} \geq \pi_{r g}=p_{g}^{w}$ for some $r \notin C$, since all agents outside the coalition are bidding $p^{w}$. Then, the corresponding term in (1) is positive or zero. Finally, if for some good $\sum_{i \in C} x_{i g}^{\prime}=0$, the corresponding term is zero. This shows that the inequality (1) above is impossible.

The logic behind Proposition 1 is the following: Suppose that market prices and the allocation are Walrasian and a group of agents, say all the oil producing countries, collude and attempt to raise the price of oil. Since importing countries make no change in their bidding strategies, VT implies that oil producing countries cannot sell a single drop of oil and thus they can not improve welfare by colluding. Since a SE is a NE, we have that:

Corollary 1. Let $(S, f)$ a game form in which VT and $\mathbf{U}$ hold. Then, if $\left(p^{w}, x^{w}\right.$, $\left.m^{w}\right)$ is a WE, $\exists$ s such that $s$ is $a \mathrm{NE}$ and $f(s)=\left(x^{w}, m^{w}\right)$.

In order to obtain a converse to Proposition 1 we need some extra conditions. First we impose two additional axioms on the class of mechanisms. Second, we 
assume that for each $i, u_{i}()$ is continuous on money, and that for each $p$, there exists a utility maximizing choice in $B_{i}(p)$. Finally, the converse to Proposition 1 applies only to active NE: A NE, $s^{n}$, is active if for each $g$, there is an agent $j$ with $x_{j g}\left(s^{n}\right)>0$.

Reactiveness (R). Let $s$ be a strategy profile such that in market $g, \pi_{i g}>\pi_{j g}$ with $x_{i g}(s)>0$ and $x_{j g}(s)<0$. Then $\exists s^{\prime}$, identical to s except in component $r$ (with $r=i$ or $r=j$ ) such that $\forall k \neq g$ :

$$
x_{r}\left(s^{\prime}\right)=x_{r}(s),\left(p_{g}\left(s^{\prime}\right)-p_{g}(s)\right) x_{r g}(s)<0 \text { and } p_{k}(s)=p_{k}\left(s^{\prime}\right)
$$

This axiom means that, when the bid made by a buyer is greater than the bid made by a seller, there is a way for (at least) one of them to modify the price (to increase the price if he is a seller, or to decrease the price if he is a buyer) without affecting either her consumption bundle or the other prices. Recall that we are interpreting $\pi^{\prime} s$ as maximum buying (resp. minimum selling) bids. Thus, Axiom $\mathbf{R}$ says that any discrepancy between those bids can be eliminated by our market game with a minimal impact on allocations and other prices. In other words, the mechanism mimics what in this circumstances is a natural reaction of maximizing agents, namely to push the market price downwards (buyers) or upwards (sellers). We remark that this axiom does not imply that the general equilibrium effects of a change in the price of good $g, p_{g}$, vanish. What it means is that when two agents want to change $p_{g}$, the market games give room to, at least, one of them to do so, leaving other prices unaffected. Thus, this axiom is automatically satisfied in general equilibrium models of price-making agents. We will now postulate the following:

Strong Bertrand Competition (SBC). Let $s_{-i}$ be a strategy profile where all active traders minus $i$ quote the same bid $p$. Given $x_{i}^{\prime}$, if $x_{i}^{\prime} \neq 0$, let $I=\{g \in G \mid$ $\left.x_{i g}^{\prime}>0\right\}$ and $D=\left\{g \in G \mid x_{i g}^{\prime}<0\right\}$. Then for all $\pi_{i}^{\prime}$ such that $\pi_{i g}^{\prime}>p_{g}$ $\forall g \in I$ and $\pi_{i g}^{\prime}<p_{g} \forall g \in D, \exists q_{i}$ such that: $x_{i}^{\prime}=x_{i}\left(\pi_{i}^{\prime}, q_{i}, s_{-i}\right)$, and $m_{i}\left(\pi_{i}^{\prime}\right.$, $\left.q_{i}, s_{-i}\right)=-p\left(\pi_{i}^{\prime}, q_{i}, s_{-i}\right) x_{i}^{\prime}$. If $x_{i}^{\prime}=0$, then there exist $s_{i}^{\prime}=\left(\pi_{i}^{\prime}, q_{i}^{\prime}\right)$ with $\pi_{i}^{\prime}=p$ such that $x_{i}^{\prime}=x_{i}\left(s_{i}^{\prime}, s_{-i}\right)$, and $m_{i}\left(s_{i}^{\prime}, s_{-i}\right)=0$.

The SBC Axiom says that a seller (resp. a buyer) by cutting (resp. increasing) the market price can transact as much as she wants. Furthermore, by agreeing in the price announcement of all the other agents he always has the possibility of no trade. This implies that in any active NE in which agents quote the same price, the outcome is individually rational. The adjective "strong" refers to the fact that in textbook Bertrand competition, by undercutting market price a firm can get all the demand at this new price. In our case, by undercutting others, an agent can get as much as she wants. Now we have the following result:

Proposition 2. In any market game form in which $\mathbf{V T}, \mathbf{R}$ and $\mathbf{S B C}$ hold any active NE yields a Walrasian allocation.

Proof. We will first prove that for any pair of active traders $i$ and $j$ in a market, say $g$, we have that in any NE $\pi_{i g}=\pi_{j g}$. Because $V T$ if the market is active and, say $x_{i g}(s)>0($ resp. $<)$, it must be that $p_{g}(s) \leq($ resp. $\geq) \pi_{i g}$. Suppose that there 
exists a pair of active traders $i$ and $j$ such that $\pi_{i g}>\pi_{j g}$ where $i$ (resp. $j$ ) is the net buyer (resp. seller). Suppose that in Axiom $R, r=i$. Then, $\exists s_{i}^{\prime}$ such that

$$
x_{i}\left(s^{\prime}\right)=x_{i}(s), p_{g}\left(s^{\prime}\right)<p_{g}(s) \text { and } p_{k}(s)=p_{k}\left(s^{\prime}\right), \forall k \neq g
$$

But then, $i$ is clearly better off by playing $s_{i}^{\prime}$ because preferences are monotonic on money. This contradicts that we are at a NE. The same argument holds if $r=$ $j$. Therefore, in an active market, any pair of a supplier and a demander announce the same price. Let $\pi_{g}^{*}$ the common price announcement in market $g$. Let $\pi^{*}=$ $\left(\pi_{g}^{*}\right)_{g \in G}$. By VT, buyer $i^{\prime} s$ price announcement $\pi_{i g}$ and seller $j^{\prime} s$ announcement $\pi_{j g}$ should satisfy $\pi_{j g} \leq p_{g}(s) \leq \pi_{i g}$. Since $\pi_{i g}=\pi_{j g}=\pi_{g}^{*}, p_{g}(s)=\pi_{g}^{*}$. Therefore, $p(s)=\pi^{*}$.

Let $\left(x_{i}, m_{i}\right)$ be the bundle obtained by $i$ in a NE and $\left(x_{i}^{\prime}, m_{i}^{\prime}\right)$ be a most preferred bundle for $i$ in $B_{i}(p(s))$ (this bundle exists by assumption). Obviously $u_{i}\left(x_{i}^{\prime}, m_{i}^{\prime}\right) \geq u_{i}\left(x_{i}, m_{i}\right)$. If $u_{i}\left(x_{i}^{\prime}, m_{i}^{\prime}\right)=u_{i}\left(x_{i}, m_{i}\right) \forall i \in H$, the NE yields a Walrasian allocation. Let us assume that there is a trader, say $i$, for whom $u_{i}\left(x_{i}^{\prime}\right.$, $\left.m_{i}^{\prime}\right)>u_{i}\left(x_{i}, m_{i}\right)$. By SBC, we know that the outcome of an active NE in which agents quote the same price is individually rational. Thus, $x_{i}^{\prime} \neq 0$. Let $I=\{g \in$ $\left.G / x_{i g}^{\prime}>0\right\}$ and $D=\left\{g \in G / x_{i g}^{\prime}<0\right\}$. Let $\pi_{i g}^{\prime}=p_{g}(s)+\epsilon$ for all $g \in I$, and $\pi_{i g}^{\prime}=p_{g}(s)-\epsilon$ for all $g \in D$. By $S B C$ there is $q_{i}$ such that $x_{i}^{\prime}=x_{i}\left(\pi_{i}^{\prime}, q_{i}, s_{-i}\right)$, and $m_{i}\left(\pi_{i}^{\prime}, q_{i}, s_{-i}\right)=-p\left(\pi_{i}^{\prime}, q_{i}, s_{-i}\right) x_{i}^{\prime}$. By $V T, p_{g}\left(\pi_{i}^{\prime}, q_{i}, s_{-i}\right) \leq \pi_{i g}^{\prime}=$ $p_{g}(s)+\epsilon$ for all $g \in I$, and $p_{g}(s)-\epsilon=\pi_{i g}^{\prime} \leq p_{g}\left(\pi_{i}^{\prime}, q_{i}, s_{-i}\right)$ for all $g \in D$. Since the outcome function selects balanced allocations, for each $g \in I$ there is an agent $j \neq i$ which is a net supplier of good $g$. That is, $x_{j g}\left(\pi_{i}^{\prime}, q_{i}, s_{-i}\right)<0$, and then, by $V T, p_{g}(s)=\pi_{j g} \leq p_{g}\left(\pi_{i}^{\prime}, q_{i}, s_{-i}\right)$. If $g \in D$, there is an agent $k \neq i$ which is a net demander of good $g$. That is, $x_{j g}\left(\pi_{i}^{\prime}, q_{i}, s_{-i}\right)>0$, and then, by $V T, p_{g}\left(\pi_{i}^{\prime}\right.$, $\left.q_{i}, s_{-i}\right) \leq \pi_{j g}=p_{g}(s)$. Then, summarizing we get that:

$$
\begin{aligned}
p_{g}(s) & \leq p_{g}\left(\pi_{i}^{\prime}, q_{i}, s_{-i}\right) \leq p_{g}(s)+\epsilon \text { for all } g \in I \\
p_{g}(s)-\epsilon & \leq p_{g}\left(\pi_{i}^{\prime}, q_{i}, s_{-i}\right) \leq p_{g}(s) \text { for all } g \in D
\end{aligned}
$$

Therefore

$$
m_{i}^{\prime} \geq m_{i}\left(\pi_{i}^{\prime}, q_{i}, s_{-i}\right) \geq m_{i}^{\prime}-\sum_{g \in I} \epsilon x_{i g}^{\prime}+\sum_{g \in D} \epsilon x_{i g}^{\prime} .
$$

Because preferences are continuous on money, for a sufficiently small $\epsilon$,

$$
u_{i}\left(x_{i}^{\prime}, m_{i}^{\prime}\right)>u_{i}\left(x_{i}^{\prime}, m_{i}\left(\pi_{i}^{\prime}, q_{i}, s_{-i}\right)\right)>u_{i}\left(x_{i}, m_{i}\right)
$$

which is a contradiction. Thus, any active NE yields Walrasian allocations.

Corollary 2. Under the conditions of Proposition 2, any Strong Equilibrium where all markets are active yields Walrasian allocations.

Theorem 1. Any market game satisfying U, VT, R, and SBC doubly implements the Walrasian correspondence in Nash and Strong equilibrium if all markets are active.

Axiom SBC implies that for some strategy profiles, the resulting allocation is not individually feasible. Individual feasibility can be restored by redefining 
SBC in such a way that the deviating agent can get any allocation compatible with individual feasibility and balancedness. In this case, the conclusion of Proposition 2 would refer to Constrained Walrasian Equilibria, defined as a feasible allocation and a vector of prices such that each agent maximizes utility subject to the budget constraint and to the feasibility constraint (i.e., no agent can demand more than the aggregate endowment). ${ }^{6}$

To close this section we describe two examples fulfilling all our axioms. The basic difference between the two examples is how people are matched when they submit different bids. In the first example, we match all the agents bidding above the market price with the agents bidding below market price. These agents can trade among them whenever there is no one bidding exactly the market price. Otherwise, they get zero. In the second example, different groups are formed depending on how big the bids are. In this case, trade is organized in a way that the group with the smallest bid above market price trade with the group with the biggest bid below market price and we continue matching different groups in this way. Finally we notice (see Sect. 4) that a modification of the market game proposed by Svensson (1991) in economies with indivisible goods also satisfies our axioms.

Example 1. Consider the class of market games, $\left(S_{i}, f\right), i \in H$, with the following properties: Given the strategy $s=(\pi, q)$, for each good $g \in G$, the price function $p_{g}(s)$ is such that: (1) For each market $g$, the final price only depends on the bids of the agents for that market, that is, $p_{g}(s)=p_{g}\left(\pi_{g}\right)$. (2) The function $p_{g}(s)$ is continuous. (3) The function $p_{g}(s)$ is strictly monotone in $\pi_{i g}$. (4) For all $g \in G$, $\min _{i \in H}\left\{\pi_{i g}\right\} \leq p_{g}(s) \leq \max _{i \in H}\left\{\pi_{i g}\right\}$. To define the allocation of goods, we distinguish among the subsets of agents $N_{g}, I_{g}$, and $D_{g}$ defined as follows:

$$
\begin{aligned}
N_{g} & =\left\{i \in H \mid \pi_{i g}=p_{g}(s)\right\} \\
I_{g} & =\left\{i \in H \mid \pi_{i g}<p_{g}(s)\right\} \\
D_{g} & =\left\{i \in H \mid \pi_{i g}>p_{g}(s)\right\}
\end{aligned}
$$

Net trades of good $g$ allocated to each of the agents in those sets only depend on $q_{g}$ and are described as follows:

(I) If $N_{g} \neq \emptyset$, for all $i \notin N_{g}, x_{i g}(s)=0$, and for all $i \in N_{g}, x_{i g}(s)$ should be such that $\sum_{i \in N_{g}} x_{i g}(s)=0$, and $x_{i g}(s)=q_{i g}$ whenever $\sum_{i \in N_{g}} q_{i g}=0$. Some examples are:

(a) For all $i \in N_{g}$, if $\left(\sum_{j \in N_{g}} q_{j g}\right) q_{i g} \leq 0$, then $x_{i g}(s)=q_{i g}$, and for the rest of agents in $N_{g}$ that does not satisfy this (let $S \subseteq N_{g}$ be such a set),

$$
x_{i g}(s)=q_{i g}-\frac{\sum_{j \in N_{g}} q_{j g}}{|S|} .
$$

(b) For all $i \in N_{g}$, if $\left(\sum_{j \in N_{g}} q_{j g}\right) q_{i g} \leq 0$, then $x_{i g}(s)=q_{i g}$, and for the rest of agents in $N_{g}$ that does not satisfy this (let $S \subseteq N_{g}$ be such a set),

$$
x_{i g}(s)=\frac{-\sum_{j \in N_{g} \backslash S} q_{j g}}{\sum_{j \in S} q_{j g}} q_{i g},
$$

\footnotetext{
${ }^{6}$ A formal proof of this statement is available under request.
} 
that is, among this agents we apply a proportional rule.

(c) If $\sum_{j \in N_{g}} q_{j g} \geq 0$, for all $i \in N_{g}$ such that $q_{i g} \leq 0, x_{i g}(s)=q_{i g}$, and for the rest of agents,

$$
x_{i g}(s)=\min \left\{\lambda, q_{i g}\right\}, \text { with } \lambda \in \mathbb{R}_{+} \text {and s.t. } \sum_{i \in N_{g}} x_{i g}(s)=0 .
$$

If $\sum_{j \in N_{g}} q_{j g} \leq 0$, for all $i \in N_{g}$ such that $q_{i g} \geq 0, x_{i g}(s)=q_{i g}$, and for the rest of agents,

$$
x_{i g}(s)=\max \left\{-\lambda, q_{i}\right\} \text {, with } \lambda \in \mathbb{R}_{+} \text {and is s.t. } \sum_{i \in N_{g}} x_{i g}(s)=0 .
$$

(d) Satisfy the announced supplies, that is, for all $i \in N_{g}$ such that $q_{i g} \leq 0$, $x_{i g}(s)=q_{i g}$ (let $M$ be the total supply in absolute terms) and then apply the uniform rule on the demand, that is, if $\sum_{q_{i g}>0} q_{i g} \geq M$,

$$
x_{i g}(s)=\min \left\{q_{i g}, \lambda\right\} \text {, with } \lambda \in \mathbb{R}_{+} \text {and s.t. } \sum_{q_{i g}>0} \min \left\{q_{i g}, \lambda\right\}=M,
$$

if $\sum_{q_{i g}>0} q_{i g}<M$

$$
x_{i g}(s)=\max \left\{q_{i g}, \lambda\right\} \text {, with } \lambda \in \mathbb{R}_{+} \text {and s.t. } \sum_{q_{i g}>0} \max \left\{q_{i g}, \lambda\right\}=M .
$$

(e) Satisfy the announced demands, that is, for all $i \in N_{g}$ such that $q_{i g} \geq 0$, $x_{i g}(s)=q_{i g}$ (let $M$ be the total demand), and then apply the uniform rule on the supply, that is, if $\sum_{q_{i g} \geq 0}\left|q_{i g}\right| \geq M$,

$$
x_{i g}(s)=\max \left\{q_{i g}, \lambda\right\} \text { with } \lambda \in \mathbb{R}_{-} \text {and s.t. } \sum_{q_{i g}<0} \max \left\{q_{i g}, \lambda\right\}=M,
$$

if $\sum_{q_{i g}<0}\left|q_{i g}\right|<M$,

$$
x_{i g}(s)=\min \left\{q_{i g}, \lambda\right\} \text { with } \lambda \in \mathbb{R}_{-} \text {and s.t. } \sum_{q_{i g}<0} \min \left\{q_{i g}, \lambda\right\}=M .
$$

(f) For all $i \in N_{g}$,

$$
x_{i g}(s)=q_{i g}-\frac{\sum_{j \in N_{g}} q_{j g}}{\left|N_{g}\right|} .
$$

(II) If $N_{g}=\emptyset$ and the set of agents is greater than two, we distinguish the following cases:

(i) If $I_{g}=\{i\}$ and $\left|D_{g}\right|>1, x_{i g}(s)=\min \left\{0, q_{i}\right\}$ and for all $j \in D_{g}$, apply any allocation rule such that $x_{j g}(s) \geq 0$, and the total demand equals to $-x_{i g}(s)$. For example, among all the agents in $D_{g}$ that announce $q_{j g} \leq 0, x_{j g}(s)=0$, and for the rest of agents in this set, we can apply the uniform rule (like in (d)) or the proportional rule among others. 
(ii) If $D_{g}=\{i\}$ and $\left|I_{g}\right|>1, x_{i g}(s)=\max \left\{0, q_{i}\right\}$ and for all $j \in I_{g}$,apply any allocation rule such that $x_{j g}(s) \leq 0$, and the total supply equals to $-x_{i g}(s)$. For example, among all agents in $I_{g}$ that announce $q_{j g} \geq 0, x_{j g}(s)=0$, and for the rest of agents in this set, we can apply the uniform rule (like in (e)) or the proportional rule among others.

(iii) In any other possible case, for all $i \in D_{g}, x_{i g}(s) \geq 0$, and for all $i \in I_{g}$, $x_{i g}(s) \leq 0$ and $\sum x_{i g}(s)=0$. For example, $x_{i g}(s)=0$ for all $i \in D_{g}$ such that $q_{i g}<0$ and for all $i \in I_{g}$ such that $q_{i g}>0$, among the rest of agents we can apply rules like in (a), (b), (c), (d) or (e).

(III) Finally, if we only have two agents, and they do not announce the same price, given the properties on the price function, we will get that $N_{g}=\emptyset$, and $I_{g}=\{i\}$, $D_{g}=\{j\}$. In this situation, $x_{i g}(s) \leq 0, x_{i g}(s)=-x_{j g}(s)$ and it should be such that agent $i$ can get any $x_{i g}<0$ if he announces an appropriate $q_{i g}$ given $q_{j g}$. And, symmetrically, agent $j$ can get any $x_{j g}>0$ if he announces an appropriate $q_{j g}$ given $q_{i g}$. For example,

$$
x_{i g}(s)=\min \left\{0, q_{i g}-\frac{q_{i g}+q_{j g}}{2}\right\} \text { and } x_{j g}(s)=\max \left\{0, q_{j g}-\frac{q_{i g}+q_{j g}}{2}\right\}
$$

Let's show that this class of games satisfies all our axioms.

- Unanimity is trivially satisfied.

- If an agent $i$ is a net demander of a good $g\left(x_{i g}(s)>0\right)$, then either $i \in N_{g}$ or $N_{g}=\emptyset$ and then $i \in D_{g}$, in any case, $p_{g}(s) \leq \pi_{i g}$. If agent $j$ is a net supplier of a good $g\left(x_{j g}(s)<0\right)$, then either $j \in N_{g}$ or $N_{g}=\emptyset$ and then $j \in I_{g}$, in any case, $p_{g}(s) \geq \pi_{j g}$. Therefore Voluntary Trade is satisfied.

- Let $s$ be a strategy profile such that in market $g, \pi_{i g}>\pi_{j g}$ with $x_{i g}(s)>0$ and $x_{j g}(s)<0$. Then, clearly $N_{g}=\emptyset, i \in D_{g}$, and $j \in I_{g}$. Let $s^{\prime}$ be identical to $s$ except in component $i$, where this agent announced the same $q_{i g}$ than in $s_{i}$, and $\pi_{i g}^{\prime}=\pi_{i g}-\varepsilon$. Since $p_{g}($.$) only depends on the bids of the agents for the market g$, it is continuous and strictly monotone in $\pi_{i g}, p_{g}\left(s^{\prime}\right)<p_{g}(s)$. Furthermore, we can choose $\varepsilon$ sufficiently small such that in the new situation $N_{g}^{\prime}=\emptyset, D_{g}^{\prime}=D_{g}$ and $I_{g}^{\prime}=I_{g}$. Thus, $\forall k \neq g, x_{i}\left(s^{\prime}\right)=x_{i}(s),\left(p_{g}\left(s^{\prime}\right)-p_{g}(s)\right) x_{i g}(s)<0$ and $p_{k}(s)=p_{k}\left(s^{\prime}\right)$. Therefore Reactiveness is satisfied.

- Notice that Strong Bertrand competition is trivially satisfied in this class of games. Under the hypothesis of the Axiom, if in a market $g$ one agent deviates from the bids announcements of the others, either we are in the situation described in (i), in (ii) or in (III). In any case, the agent who deviates can transact as much as he wants. Furthermore, if he wants to stay inactive, he can always quote the same price as the others, and a $q_{i}^{\prime}$ such that he gets cero. For example, in (a),(b), (c),(d) and (e), announcing $q_{i}^{\prime}=0$, he gets cero. In (f) he gets cero if he announces $q_{i g}^{\prime}=\frac{1}{\left|N_{g}\right|-1} \sum_{j \neq i} q_{j g}$ for each $g$.

Example 2. Let $\left(S_{i}, f\right), i \in H$, be a market game form described as follows:

Given the strategy $s=(\pi, q)$, for each good $g \in G$, let $p_{g}(s)=\frac{\sum_{i \in H} \pi_{i g}}{h} .{ }^{7}$ Let

${ }^{7}$ For simplicity we consider here a very simple example of a price function. However, it can be shown that any price function satisfying properties (1) - (4) spelled out at the beginning of Example 1 yields the same result. 
$N_{g}, I_{g}$, and $D_{g}$ be subsets of agents defined as follows:

$$
\begin{aligned}
N_{g} & =\left\{i \in H \mid \pi_{i g}=p_{g}(s)\right\} \\
I_{g} & =\left\{i \in H \mid \pi_{i g}<p_{g}(s)\right\} \\
D_{g} & =\left\{i \in H \mid \pi_{i g}>p_{g}(s)\right\}
\end{aligned}
$$

Net trades of good $g$ allocated to each of the agents in those sets are:

1. For all $i \in N_{g}, x_{i g}(s)=q_{i g}-\frac{\sum_{j \in N_{g}} q_{j g}}{\left|N_{g}\right|}$. Thus, agents in $N_{g}$ trade among themselves.

2. Agents in $I_{g}$ trade with agents in $D_{g}$. We start matching those agents in $I_{g}$ with bids closer to $p_{g}(s)$ with agents in $D_{g}$ with bids also close to $p_{g}(s)$. These agents trade among themselves, and we continue matching agents from these two sets in this way. To formally describe this process, let's define the following subsets of agents.

$$
\begin{aligned}
& I_{g}^{1}=\left\{i \in I_{g} \mid q_{i g}=\min \left\{q_{j g} \mid \pi_{j g}=\max _{k \in I_{g}} \pi_{k g}\right\}\right\} \\
& D_{g}^{1}=\left\{i \in D_{g} \mid q_{i g}=\max \left\{q_{j g} \mid \pi_{j g}=\min _{k \in D_{g}} \pi_{k g}\right\}\right\} \\
& I_{g}^{k}=\left\{i \in I_{g} \backslash \cup_{l=1}^{k-1} I_{g}^{l} \mid q_{i g}=\min \left\{q_{j g} \mid \pi_{j g}=\max _{k \in I_{g} \backslash \cup_{l=1}^{k-1} I_{g}^{l}} \pi_{k g}\right\}\right\} \\
& D_{g}^{k}=\left\{i \in D_{g} \backslash \cup_{l=1}^{k-1} D_{g}^{l} \mid q_{i g}=\max \left\{q_{j g} \mid \pi_{j g}=\min _{k \in D_{g} \backslash \cup_{l=1}^{k-1} D_{g}^{l}} \pi_{k g}\right\}\right\}
\end{aligned}
$$

Agents in $I_{g}^{k}$ will trade with agents in $D_{g}^{k}$ according to the following rule:

For all $i \in I_{g}^{k}, x_{i g}(s)=\min \left\{0, q_{i g}-\frac{\sum_{j \in I_{g}^{k} \cup D_{g}^{k}} q_{j g}}{\left|I_{g}^{k} \cup D_{g}^{k}\right|}\right\}$, and for all $i \in D_{g}^{k}$, $x_{i g}(s)=\max \left\{0, q_{i g}-\frac{\sum_{j \in I_{g}^{k} \cup D_{g}^{k}} q_{j g}}{\left|I_{g}^{k} \cup D_{g}^{k}\right|}\right\}$. Notice that all agents in the same group announce the same net trade, therefore if for an agent $j \in I_{g}^{k}, x_{j g}(s)=0$, then for all $i \in I_{g}^{k}, x_{i g}(s)=0$ and for all agent $i \in D_{g}^{k}, x_{i g}(s)=0$ and vice versa. Therefore, final net trades among these groups are balanced, that is, $\sum_{i \in I_{g}^{k} \cup D_{g}^{k}} x_{i g}(s)=0$. Notice also that if one of these sets is empty, there is no trade. That is, suppose that for some $k, I_{g}^{k}=\emptyset$, then for all $i \in D_{g}^{k}, x_{i g}(s)=0$.

Let's show that this market game satisfies all our axioms.

- Unanimity is trivially satisfied.

- Notice that if $x_{i g}(s)>0, i \in N_{g} \cup D_{g}^{k}$ for some $k$, and then $\pi_{i g} \geq p_{g}(s)$. If $x_{i g}(s)<0, i \in N_{g} \cup I_{g}^{k}$ for some $k$, and then $\pi_{i g} \leq p_{g}(s)$. Therefore Voluntary Trade is satisfied.

- Suppose that $s$ is a strategy profile such that in market $g, \pi_{i g}>\pi_{j g}$ with $x_{i g}(s)>0$ and $x_{j g}(s)<0$. Then $\pi_{j g} \leq p_{g}(s)<\pi_{i g}$ or $\pi_{j g}<p_{g}(s) \leq \pi_{i g}$. Let suppose first that $\pi_{j g} \leq p_{g}(s)<\pi_{i g}$. In this case we have to show that 
there is a way for agent $i$ to reduce the price of good $g$ without affecting her consumption bundle. In order to guarantee this, let $s^{\prime}$ be a strategy identical to $s$ except in component $i g$. Let $\pi_{i g}^{\prime}$ be such that the mean of the bids is an $\varepsilon$ less than $\pi_{i g}^{\prime}$. Thus, $p_{g}\left(s^{\prime}\right)<\pi_{i g}^{\prime}$, which implies that there is at least one agent $j$ such that $\pi_{j g}<p_{g}\left(s^{\prime}\right)$, and therefore, $I_{g}^{1} \neq \emptyset$. We can choose $\varepsilon$ sufficiently small such that $D_{g}^{1}=\{i\}$. Then agent $i$ will trade, according to our mechanism, with the agents in $I_{g}^{1}$. In order to obtain the same net trade than agent $i$ gets under the strategy $s$, let $q_{i g}^{\prime}$ be such that $x_{i g}(s)=q_{i g}^{\prime}-\frac{q_{i g}^{\prime}+\sum_{j \in I_{g}^{1}} q_{j g}}{\left|I_{g}^{1}\right|+1}$. Finally, we have to show that $p_{g}\left(s^{\prime}\right)<p_{g}(s)$. For this, we only need to prove that $\pi_{i g}^{\prime}<\pi_{i g}$. Since $p_{g}\left(s^{\prime}\right)=\pi_{i g}^{\prime}-\varepsilon=\frac{\pi_{i g}^{\prime}+\sum_{j \neq i} \pi_{j g}}{h}$, we have that $(h-1) \pi_{i g}^{\prime}=$ $h p_{g}(s)-\pi_{i g}+h \varepsilon$. Since $p_{g}(s)<\pi_{i g}$, we can always choose $\varepsilon$ sufficiently small such that $\pi_{i g}^{\prime}<\pi_{i g}$. Thus, given this $s^{\prime}, p_{g}\left(s^{\prime}\right)<p_{g}(s)$ and $x_{i g}\left(s^{\prime}\right)=x_{i g}(s)$. For the case where $\pi_{j g}<p_{g}(s) \leq \pi_{i g}$, we can, in a similar way as above, construct a strategy for agent $j$ such that $p_{g}\left(s^{\prime}\right)>p_{g}(s)$ and $x_{j g}\left(s^{\prime}\right)=x_{j g}(s)$. Therefore Reactiveness is satisfied.

- Let $s_{-i}$ be a strategy profile where all active agents minus $i$ quote the same bid $p$. Given $x_{i}^{\prime} \neq 0$, let $I=\left\{g \in G \mid x_{i g}^{\prime}>0\right\}$ and $D=\left\{g \in G \mid x_{i g}^{\prime}<0\right\}$. For each $g \in I$, and for all $\pi_{i g}^{\prime}>p_{g}$, let $q_{i g}^{\prime}$ be such that $x_{i g}^{\prime}=q_{i g}^{\prime}-\frac{q_{i g}^{\prime}+\sum_{j \in I_{g}^{1}} q_{j g}}{\left|I_{g}^{1}\right|+1}$. The net trade $q_{i g}^{\prime}$ is well defined because $p_{g}<p_{g}\left(s^{\prime}\right)<\pi_{i g}$ and therefore $I_{g}^{1} \neq \emptyset$. For each $g \in D$, and for all $\pi_{i g}^{\prime}<p_{g}$, let $q_{i g}^{\prime}$ be such that $x_{i g}^{\prime}=$ $q_{i g}^{\prime}-\frac{q_{i g}^{\prime}+\sum_{j \in D_{g}^{1}} q_{j g}}{\left|D_{g}^{1}\right|+1}$. The net trade $q_{i g}^{\prime}$ is well defined because $D_{g}^{1} \neq \emptyset$. Given the strategy $s=\left(s_{-i}, s_{i}^{\prime}\right)$ with $s_{i}^{\prime}=\left(\pi^{\prime}, q^{\prime}\right)$, for each $g \in G, x_{i g}\left(s^{\prime}\right)=x_{i g}^{\prime}$. If $x_{i}^{\prime}=0$, then by announcing $\pi_{i}^{\prime}=p$, all agents are in $N_{g}$, and by announcing

$q_{i}^{\prime}=\frac{1}{\left|N_{g}\right|-1} \sum_{j \neq i} q_{j g}$ for each $g$, he gets cero. Therefore Strong Bertrand Competition is satisfied.

\section{Relationship with the literature}

We end the paper by discussing the relation of our results with those obtained by Svensson (1991) and Benassy (1986).

In the market game proposed by Svensson (1991) for economies with indivisible goods, all active and $t i g h t^{8}$ Nash equilibria are strong equilibria that yield Walrasian allocations, but there may be Nash equilibria that are not tight because reactiveness is not satisfied. This is because in Svensson's mechanism, every agent announces a price vector, an object is assigned to the agent who announced the highest price, and this is the price paid for the object. In case of a tie the only requirement is that the object is not assigned to the owner. Suppose that the bid made by a buyer is larger than the bid made by a seller, and there is a different agent who announces the same bid as the buyer. In this situation the buyer (the seller) cannot decrease (increase) the price without affecting his consumption. However, Svensson's game

\footnotetext{
8 A Nash equilibrium is tight if the seller's and the buyer's bids are equal.
} 
can be easily modified in order to satisfy reactiveness: Every agent announces a price vector, an object is assigned to the agent who announces the highest price, and the price paid for the object is the one announced by the owner of the object. This modified mechanism is another example of a mechanism which satisfies all our axioms.

With respect to the Benassy paper (1986) it can be easily established that there is a close connection between his and our axioms. Our VT axiom is similar to Benassy's Assumption 1. The role played by $\mathbf{R}$ in our paper is played in Benassy by his Assumptions 2, 3, 5 and his "market by market" restriction (p. 97). In fact, it is not difficult to show that Benassy" $s$ assumptions imply $\mathbf{R}$.

\section{References}

Benassy, J. P. (1986) On competitive market mechanisms. Econometrica 54: 95-108

Demange, G., Gale, D. (1985) The strategy structure of two-sided matching markets. Econometrica 53: $873-888$

Dubey, P. (1982) Price-quantity strategic market games. Econometrica 50: 111-126

Gale, D. (1984) Equilibrium in a discrete exchange economy with money. International Journal of Game Theory 13: 61-64

Hurwicz, L. (1979) On allocations attainable through Nash equilibria. Journal of Economic Theory 21, $140-165$

Kaneko, M. (1983) Housing market with indivisibilities. Journal of Urban Economics 13: 22-50

Maskin, E. (1987) On the fair allocation of indivisible goods. In: Feiwell, G. (ed.) Arrow and the foundations of the theory of public policy. MacMillan Press, London, pp. 343-349

Postlewaite, A., Wettstein, D. (1989) Feasible and continuous implementation. Review of Economic Studies 56: 603-611

Quinzii, M. (1984) Core and equilibria with indivisibilities. International Journal of Game Theory 13: 41-61

Roth, A. E. (1982) Incentives compatibility in a market with indivisible goods. Economic Letters 9: $127-132$

Roth, A. E., Sotomayor, M. (1988) Interior points in the core of two-sided matching markets. Journal of Economic Theory 45: 85-101

Schmeidler, D. (1980) Walrasian analysis via strategic outcome functions. Econometrica 48: 1585-1593

Shubik, M. (1977) A price-quantity buy-sell market with or without contingent bids. Cowles Foundation Discussion Paper

Silvestre, J. (1985) Voluntary and efficient allocations are Walrasian. Econometrica 53: 807-816

Svensson, L.-G. (1984) Competitive equilibria with indivisible goods. Zeitschrift für Nationalokonomie 44: $375-386$

Svensson, L.-G. (1991) Nash implementation of competitive equilibria in a model with indivisible goods. Econometrica 59(3): 869-877

Tadenuma, K., Thomson, W. (1990) No-envy, consistency and monotonicity in economies with indivisible goods. Econometrica 59(6): 1755-1767

Tian, G. (1992) Implementation of the Walrasian correspondence without continuous, convex and ordered preferences. Social Choice and Welfare 9: 117-130 